\title{
Sensor-Based Interactive Balance Training with Visual Joint Movement Feedback for Improving Postural Stability in Diabetics with Peripheral Neuropathy: A Randomized Controlled Trial
}

\author{
Gurtej Singh Grewal ${ }^{a, b}$ Michael Schwenk ${ }^{a, b}$ Jacqueline Lee-Eng ${ }^{a, b}$ \\ Saman Parvaneh ${ }^{a, b}$ Manish Bharara ${ }^{b}$ Robert A. Menzies ${ }^{c}$ Talal K. Talal $^{c}$ \\ David G. Armstrong ${ }^{\text {b }}$ Bijan Najafia ${ }^{a}$ b \\ a Interdisciplinary Consortium on Advanced Motion Performance (iCAMP), and b Southern Arizona Limb Salvage \\ Alliance (SALSA), Department of Surgery, College of Medicine, University of Arizona, Tucson, Ariz., USA; ${ }^{\circ} \mathrm{Hamad}$ \\ Medical Co., Doha, Qatar
}

\author{
Key Words \\ Exergaming $\cdot$ Body-worn sensors $\cdot$ Diabetes $\cdot$ Postural \\ balance Exercise
}

\begin{abstract}
Background: Individuals with diabetic peripheral neuropathy (DPN) have deficits in sensory and motor skills leading to inadequate proprioceptive feedback, impaired postural balance and higher fall risk. Objective: This study investigated the effect of sensor-based interactive balance training on postural stability and daily physical activity in older adults with diabetes. Methods: Thirty-nine older adults with DPN were enrolled (age $63.7 \pm 8.2$ years, BMI $30.6 \pm 6,54 \%$ females) and randomized to either an intervention (IG) or a control (CG) group. The IG received sensor-based interactive exercise training tailored for people with diabetes (twice a week for 4 weeks). The exercises focused on shifting weight and crossing virtual obstacles. Body-worn sensors were implemented to acquire kinematic data and provide real-time joint visual feedback during the training. Outcome measurements included changes in center of mass (CoM) sway, ankle
\end{abstract}

and hip joint sway measured during a balance test while the eyes were open and closed at baseline and after the intervention. Daily physical activities were also measured during a 48-hour period at baseline and at follow-up. Analysis of covariance was performed for the post-training outcome comparison. Results: Compared with the CG, the patients in the IG showed a significantly reduced CoM sway $(58.31 \%$; $p=0.009)$, ankle sway $(62.7 \% ; p=0.008)$ and hip joint sway (72.4\%; $p=0.017$ ) during the balance test with open eyes. The ankle sway was also significantly reduced in the IG group (58.8\%; $p=0.037$ ) during measurements while the eyes were closed. The number of steps walked showed a substantial but nonsignificant increase $(+27.68 \% ; p=0.064)$ in the IG following training. Conclusion: The results of this randomized controlled trial demonstrate that people with DPN can significantly improve their postural balance with diabetesspecific, tailored, sensor-based exercise training. The results promote the use of wearable technology in exercise training; however, future studies comparing this technology with commercially available systems are required to evaluate the benefit of interactive visual joint movement feedback.

(c) 2015 S. Karger AG, Basel

\begin{tabular}{ll}
\hline KARGER 125/s & $\begin{array}{l}\text { @ 2015 S. Karger AG, Basel } \\
0304-324 X / 15 / 0616-0567 \$ 39.50 / 0 \quad \text { Open carccess }\end{array}$ \\
E-Mail karger@karger.com & $\begin{array}{l}\text { This is an Open Access article licensed under the terms of the } \\
\text { www.karger.com/ger }\end{array}$ \\
$\begin{array}{l}\text { Creative Commons Attribution-NonCommercial 3.0 Un- } \\
\text { ported license (CC BY-NC) (www.karger.com/OA-license), } \\
\text { applicable to the online version of the article only. Distribu- } \\
\text { tion permitted for non-commercial purposes only. }\end{array}$
\end{tabular}

Bijan Najafi, $\mathrm{PhD}$

Interdisciplinary Consortium on Advanced Motion Performance (iCAMP)

Department of Surgery, College of Medicine, University of Arizona

1501 N Campbell Ave No. 4325, Tucson, AZ 85724 (USA)

E-Mail najafi.bijan@gmail.com 


\section{Introduction}

Diabetic peripheral neuropathy (DPN), a common complication of type 2 diabetes mellitus, affects up to $50 \%$ of all people with diabetes [1] and increases the risk of foot ulceration and amputation [2,3]. Deficits in sensory and motor skills lead to inadequate proprioceptive feedback, impaired postural balance and high fall risk [4-7]. The fall-related injury risk is 15 times greater in this population compared to age-matched healthy adults [8]. Interestingly, most people suffering from distal sensorimotor polyneuropathy may not be aware of it [9]; and therefore, living with such conditions can significantly increase the fall risk in this population.

Benefits of exercise training in patients with diabetes have previously been demonstrated $[10,11]$; however, conventional training may not always be appropriate for DPN patients due to their higher fall risk, lost joint perception and reduced joint mobility, which may limit the accuracy of exercise performance [4, 7]. Further, there is a risk of excessive plantar loading during exercise which has been associated with a high risk of foot ulcers [12]. Several factors need to be considered during the development and implementation of exercise for DPN patients including the compensation of lost joint perception, controlling the intensity of training to avoid overtaxing and the personalization of exercise to meet DPN patients' needs. With advancements in technology, exergaming and virtual reality techniques have been evaluated for the training of motor control in the elderly $[13,14]$, with benefits including the concordance of visual and proprioceptive information, salient feedback from joint movement as well as the activation of motor-related areas in the brain $[15,16]$.

The current study evaluated the effectiveness of a tailored sensor-based interactive exercise with real-time joint feedback from the lower extremities in improving postural stability and daily physical activities in DPN patients. The exercise training specifically focuses on the motion range of the ankle joint and the lower extremities. We hypothesize that exercise training through an interactive interface with real-time visual joint movement during exercise will improve postural stability.

\section{Methods}

\section{Study Design}

A single-blinded, randomized, controlled trial with two arms, an intervention group (IG) and a control group (CG), was conducted to evaluate the effectiveness of a 4 -week sensor-based bal- ance training with real-time visual feedback from the joint motion of the lower extremities to improve the postural stability and activity level. Randomization was done using a computer-generated list. Allocation was concealed from the staff using opaque envelopes. Both groups received standard of care. All of the participants signed a written consent approved by the local Institutional Review Board at the University of Arizona in Tucson, Ariz., USA, and Hamad Medical Co. in Doha, Qatar.

\section{Study Population}

A sample of 54 patients with DPN were screened and 39 (age 51-81 years) were recruited from outpatient clinics in Tucson, Ariz., USA, and Doha, Qatar (fig. 1). A qualified member of staff screened the participants according to the following inclusion criteria: the ability to walk independently for $20 \mathrm{~m}$ and medically diagnosed type 2 diabetes with peripheral neuropathy. Peripheral neuropathy was confirmed using the criteria explained in the American Diabetes Association statement and the insensitivity to $10 \mathrm{~g}$ Semmes-Weinstein monofilament [17]. Additionally, the vibration perception threshold (VPT) score was recorded to quantify the level of neuropathy with a cutoff of $25 \mathrm{~V}$ as an indicator of neuropathy at recommended plantar foot sites. Subjects were excluded if diagnosed with cognitive, vestibular or central neurological dysfunction, musculoskeletal abnormality, active foot ulcers, Charcot's joints or a history of balance disorder unrelated to DPN. After randomization, 19 patients were in the IG and 20 in the CG.

\section{Equipment}

Using validated body-worn sensor technology (LegSys ${ }^{\mathrm{TM}}$; BioSensics LLC, Cambridge, Mass., USA), joint kinematic data were collected and processed for the assessment of balance as well as for balance training [18]. LegSys consists of 5 inertial sensors providing triaxial accelerometer, gyroscope and magnetometer data along with quaternion parameters, ideal for the estimation of 3-dimensional joint angles and position [19]. The data were acquired and transmitted at $100 \mathrm{~Hz}$ to facilitate real-time visual feedback in a virtual environment. The sensors were mounted on different body segments including the shank, thigh and lower back (fig. 2).

\section{Intervention}

Balance training exercises were designed to teach participants a better control of the movement of the center of mass (CoM) and the coordination of the lower extremities. The training included a point-to-point ankle-reaching task and a virtual obstacle-crossing task with appropriate audio-video feedback. LegSys kinematic data were processed in real-time using MatLab ${ }^{\circledR} 2013 \mathrm{~b}$ and Psychtoolbox version 2.54 and translated through an interactive game-like virtual reality interface on a 24 -inch monitor. The real-time joint feedback is intended to compensate impaired joint perception and for an easy visualization of motor errors during exercise [20]. Participants randomized into the IG group came to the outpatient clinic and participated in a 45 -min training session (including breaks) twice a week for 4 weeks. A qualified research member of staff was present during each session in order to prevent any accidents.

\section{Ankle-Reaching Task}

The aim of the ankle-reaching task was to help patients learn how to shift weight and coordinate the movements of the proximal (hip) and distal (ankle) joints, which are imperative for patients with DPN. This task has been implemented previously [21] and 


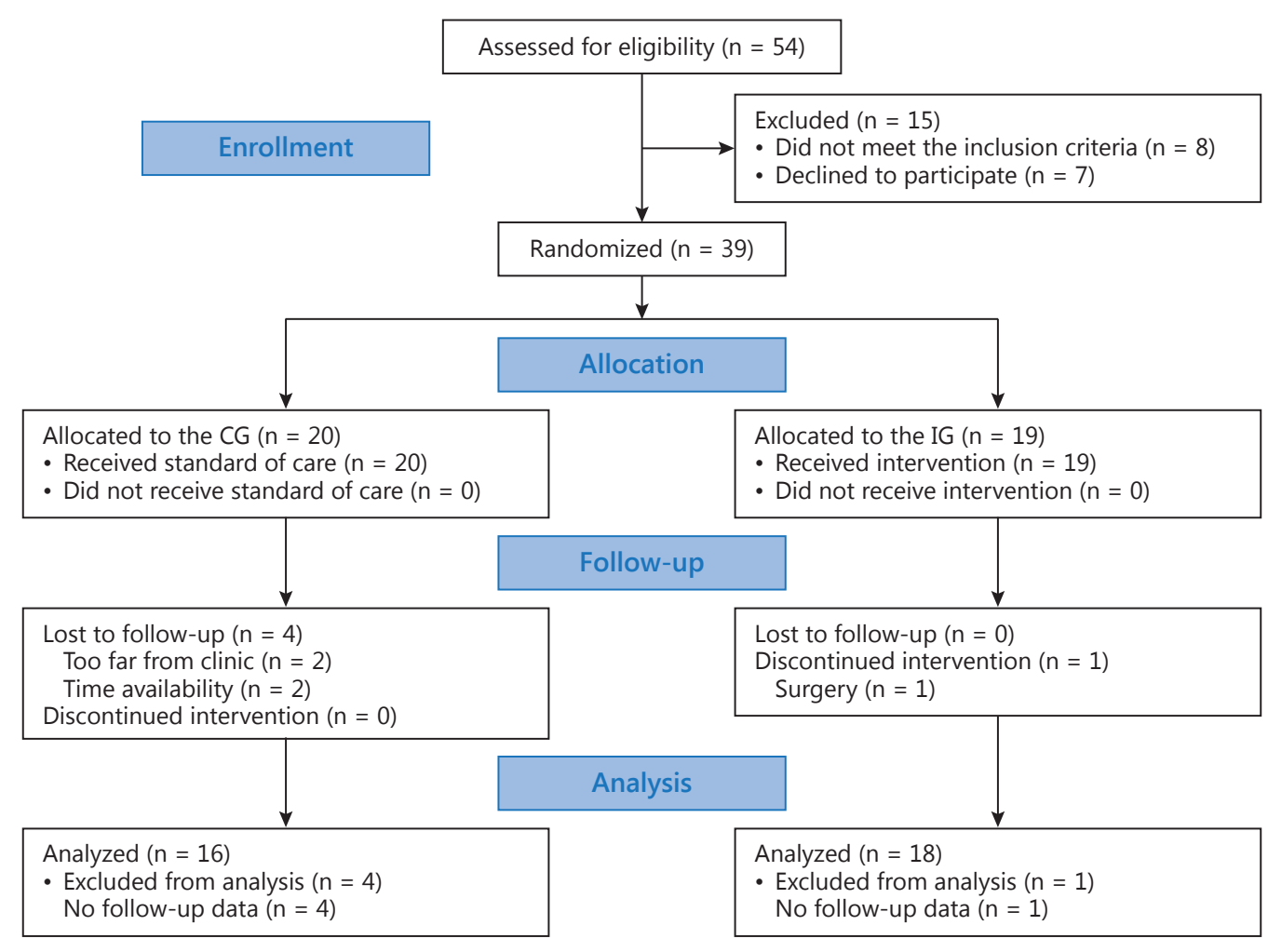

Fig. 1. CONSORT flow diagram for the study.

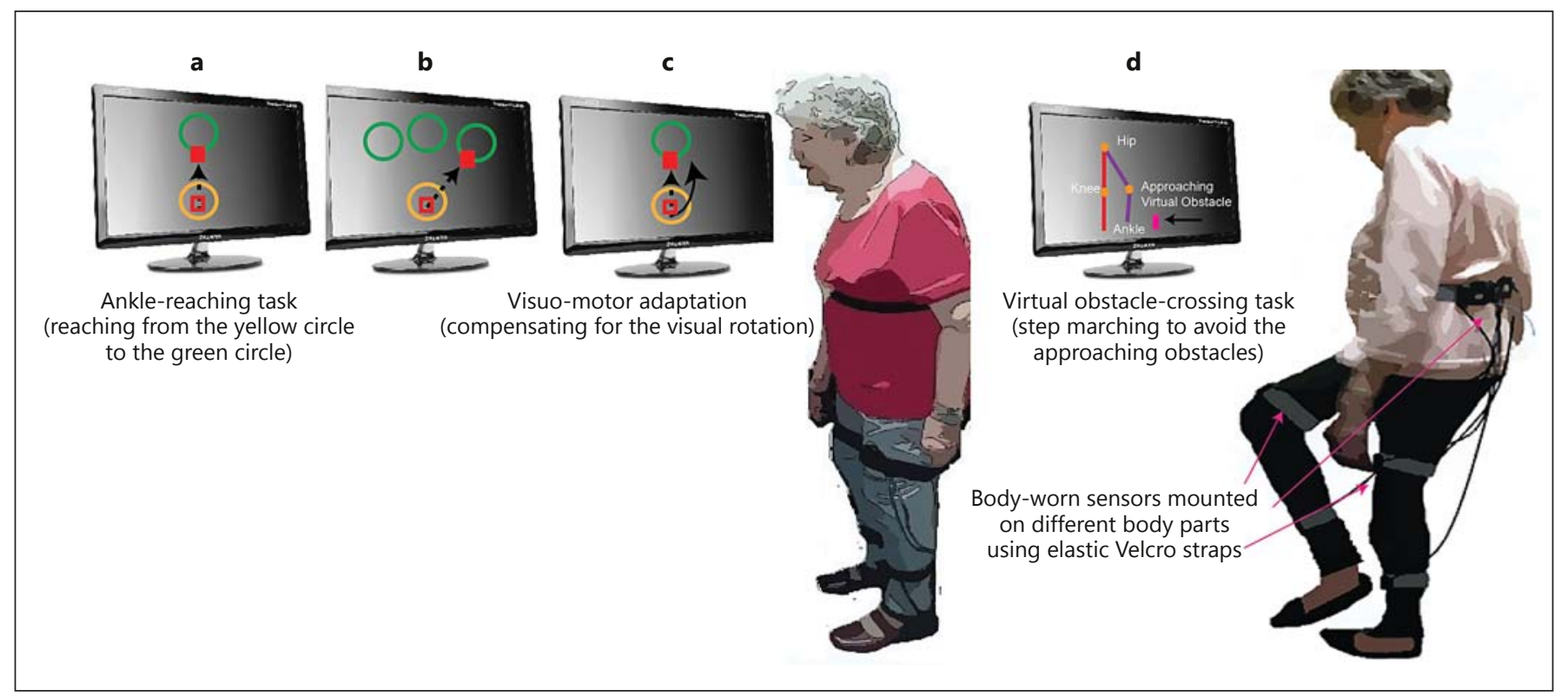

Fig. 2. An illustration of sensor-based exercise training including an ankle-reaching task, visuo-motor adaptation and a virtual obstaclecrossing task through an interactive interface. The participants per- formed exercises in front of a monitor (24 inches) providing realtime joint feedback as a dot in the ankle-reaching task and a lower extremity stick model during the virtual obstacle-crossing task. 
encourages sideward leaning and partial weight shifting that has been associated with improved postural balance [13, 22]. Kinematic data related to the rotation around the ankle joint were acquired from a shank-mounted sensor and translated into a linear movement on the computer screen. Two circles appeared sequentially on the screen (start circle in yellow followed by target circle in green; fig. 2a). After a visual start signal, the participants navigated a large red cursor from the start circle to the target circle by making a rotation around the ankle joint. The participants stood upright (always in double stance) and were instructed to move their hips in the anterior-posterior (AP) direction in order to generate dorsiflexion/ plantarflexion at the ankle without lifting the heel or toes (to move the cursor forward and backward between the circles). Similarly, a medial-lateral (ML) hip movement navigated the cursor sideward (fig. 2b). The exercise was repeated from the target to the start circle to complete one cycle of an ankle-reaching task. The participants were expected to move rapidly $(<1 \mathrm{~s})$ and accurately (navigate the cursor in the middle of the circle) from circle to circle. Upon reaching the circle in $<1 \mathrm{~s}$, the participants were awarded for the correct execution of the task with visual (the circle explodes) and audio (positive sound) feedback. If they were moving too slowly ( $>1 \mathrm{~s}$ ), the participants received visual feedback informing them about the incorrect execution of the task (the circle changed its color).

Each training session consisted of a total of 6 blocks with 20 cycles each. Training blocks 1 and 2 focused on ankle reaching in the AP direction (fig. 2a). Blocks 3 and 4 included ankle reaching in a combined AP-ML direction (fig. 2b). In order to increase the challenge, the final two blocks ( 5 and 6 ) were conducted with visuomotor rotation to help patients learn adaptation techniques [23], an aspect of particular importance for DPN patients with lost joint perception. The trajectory of the cursor representing the motion of the ankle joint was rotated to the right by $20^{\circ}$ during the visuomotor task (fig. 2c). The participants were expected to observe this change in trajectory during the reaching task and compensate for it by adjusting the ankle coordination in order to move the cursor to the target location in a point-to-point straight line. On average, each training block took $<5$ min with a 1 -min break between successive blocks in order to avoid fatigue.

\section{Virtual Obstacle-Crossing Task}

The virtual obstacle-crossing task was relatively more challenging and indulged the participants in a virtual interface, where they crossed a series of approaching virtual obstacles on the screen by step marching (fig. 2d). Kinematic data from the lower extremities were acquired from all 5 inertial sensors and translated to a simple stick model of the lower extremities displayed on the screen in real time. The stick model replicated the movements of the participants, such as lifting the foot to different heights in order to cross an approaching obstacle [24]. Different obstacle heights were practiced including 5, 10 and $15 \%$ of the length of the leg. This approach provided a safe training environment, as there were no real objects which could have caused tripping. For each obstacle height, 10 obstacles approached, one after the other, and the participants crossed them alternatively with the left or the right foot. The next virtual obstacle was released only after the participant had either crossed or hit the previous obstacle and was now in double-stance support for at least $2 \mathrm{~s}$ (for safety). The speed of the approaching obstacles was set between 0.25 and $0.5 \mathrm{~m} / \mathrm{s}$, which was translated to pixel speed on screen. Appropriate audio feedback was given upon crossing or hitting an obstacle.
Table 1. Baseline characteristics of the participants

\begin{tabular}{llll}
\hline Parameters & $\begin{array}{l}\text { CG } \\
(\mathrm{n}=16)\end{array}$ & $\begin{array}{l}\text { IG } \\
(\mathrm{n}=19)\end{array}$ & $\begin{array}{l}\mathrm{p} \\
\text { value }\end{array}$ \\
\hline Age, years & $64.90 \pm 8.50$ & $62.58 \pm 7.98$ & 0.386 \\
Females, n (\%) & $8(50)$ & $11(57.9)$ & 0.751 \\
BMI & $29.58 \pm 4.24$ & $31.78 \pm 7.53$ & 0.264 \\
Diabetes, years & $17.40 \pm 9.42$ & $17.17 \pm 10.08$ & 0.942 \\
Hemoglobin A 1 , mmol/mol & $65.40 \pm 29.91$ & $65.23 \pm 19.65$ & 0.986 \\
Mean VPT score, V & $33.52 \pm 6.16$ & $34.28 \pm 8.16$ & 0.750 \\
SF-12 & & & \\
$\quad \begin{array}{llll}\text { Physical component score } \\
\quad \text { Mental component score }\end{array}$ & $37.50 \pm 9.81$ & $37.62 \pm 10.36$ & 0.969 \\
\multicolumn{1}{|l}{ FES-I score } & $35.40 \pm 11.47$ & $32.32 \pm 12.34$ & 0.424 \\
\hline
\end{tabular}

Values are given as means \pm SD unless indicated otherwise.

\section{Measurements}

The outcome was measured at baseline and after 4 weeks using validated tests for postural stability and daily physical activities. Clinical measurements including the falls efficacy scale (FES-I) and quality of life by the short-form health survey (SF-12) were also assessed at baseline and at follow-up. Additional baseline measurements included the VPT score, hemoglobin $\mathrm{A}_{1 \mathrm{c}}$ level $(\mathrm{mmol} / \mathrm{mol})$, years of diabetes and history of fall.

\section{Balance}

Postural stability was assessed barefoot in double stance during $30 \mathrm{~s}$ with open and closed eyes using a two-link biomechanical model. The assessment has been previously validated and published in the literature $[18,21]$. The CoM was quantified from AP sway (in $\mathrm{cm}$ ), ML sway (in $\mathrm{cm}$ ) and total sway ( $\mathrm{AP} \times \mathrm{ML}$ in $\left.\mathrm{cm}^{2}\right)$. Ankle and hip sway was quantified in units of degree ${ }^{2}$. The participants stood in the upright position, keeping their feet close together but not touching. A member of the research team was present during the entire assessment period for patient safety. The test was stopped when a participant lost their balance during the procedure.

\section{Daily Physical Activities}

Physical activity was recorded using a validated T-shirt-embedded body-worn sensor (PAMSys ${ }^{\mathrm{TM}}$; BioSensics LLC) [25] in a subsample of 25 patients (CG, $n=12 ; \mathrm{IG}, \mathrm{n}=13$ ) at baseline and after 4 weeks. The system is capable of quantifying several activities and postural events. The current study was interested in the duration of sitting, standing and walking and the total number of steps taken. PAMSys was placed in the chest pocket of a custom-made T-shirt. The shirt was worn for $48 \mathrm{~h}$ and then returned via a prepaid mailing envelope or during the next clinical visit.

\section{Sample Size Justification}

Our sample size was calculated for a training-related reduction in CoM sway during balance tests with open eyes based on the results of a previous study on DPN patients [26]. The CoM sway was reduced from $3.1 \pm 2.92$ to $1.25 \pm 1.32 \mathrm{~cm}^{2}$ after 4 weeks of training. For an effect size (d) of 0.73 , a significance level of 0.05 , a power of $80 \%$ and a drop-out rate of $10 \%$, a sample size of 38 patients (19 per group) was required to verify a significant effect. 
Table 2. Effect of an interactive sensor-based exercise training on measured outcome parameters

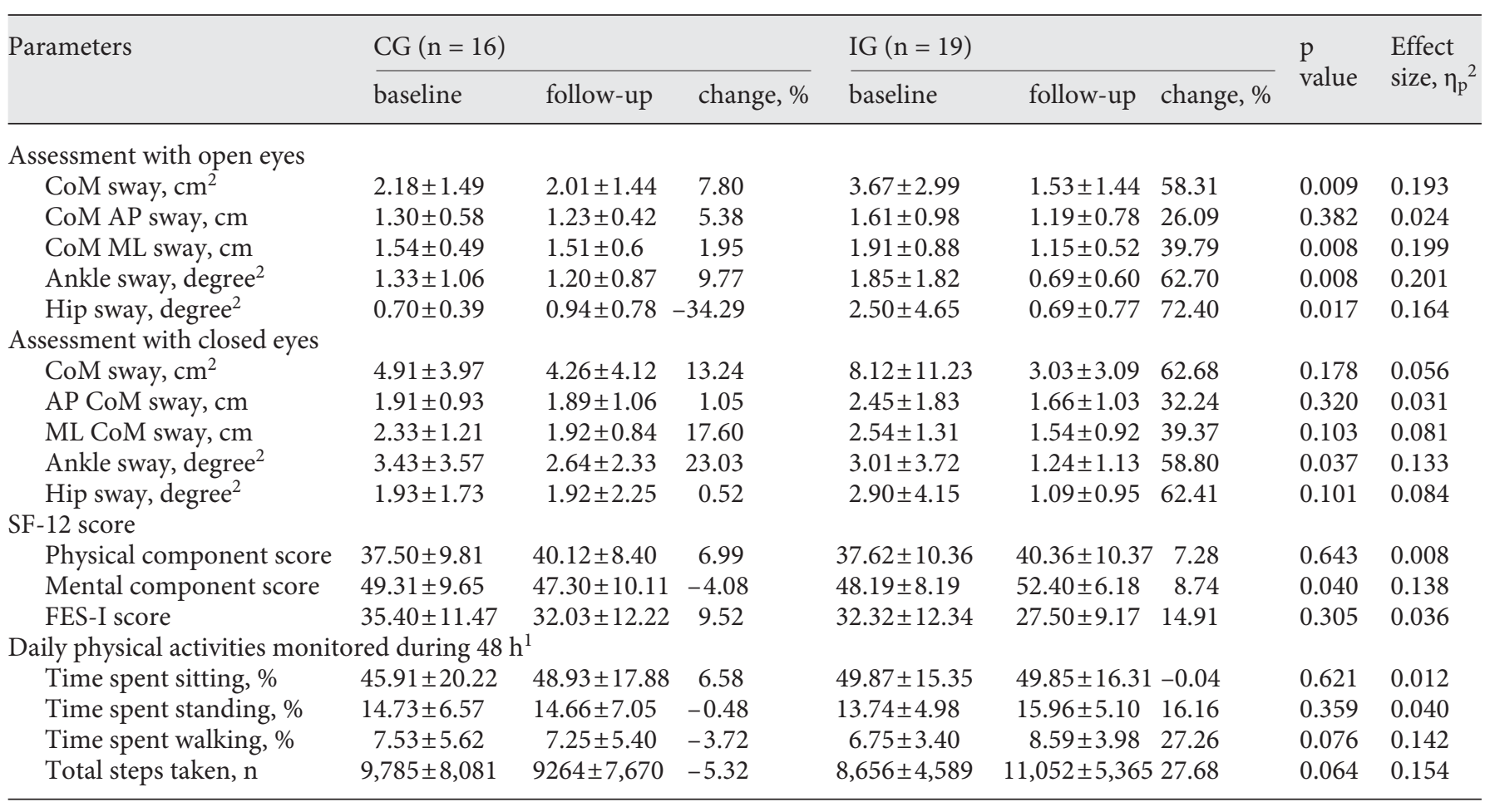

Values are given as means \pm SD. Positive change represents improvements.

${ }^{1}$ Physical activity was monitored in a subgroup of participants (CG, $\mathrm{n}=12$; IG, $\mathrm{n}=13$ ).

\section{Statistical Analysis}

All statistical analyses were performed using IBM $^{\circledR}$ SPSS version 21 (IBM, Armonk, N.Y., USA). Baseline group differences were compared by an independent $t$ test $(p<0.05$ was considered significant). The effect of training was evaluated using analysis of covariance comparing the groups' follow-up measurements adjusted to their baseline measurements ( $p<0.05$ was considered significant). The effect sizes were obtained from analysis of covariance as partial eta ${ }^{2}\left(\eta_{\mathrm{p}}{ }^{2}\right)$ with values between 0.01 and 0.06 indicating small, those between 0.06 and 0.25 indicating medium and those $>0.25$ indicating large effects [27]. Associations between baseline parameters and baseline CoM sway as well as preto post-training changes in CoM were evaluated by Pearson's correlation.

\section{Results}

Thirty-nine DPN patients were enrolled (age 63.7 \pm 8.2 years, BMI $30.6 \pm 6,54 \%$ females). Five patients dropped out of the study: 1 patient in the IG had surgery (having completed 6 training sessions) and 4 patients in the CG dropped out because of reasons related to work (time or distance), so we could not make follow-up measure- ments. No adverse events related to our balance training were reported.

The average duration of diabetes was $17.3 \pm 9.6$ years, with an average VPT score of $33.8 \pm 7.1 \mathrm{~V}$ and a hemoglobin $\mathrm{A}_{1 \mathrm{c}}$ level of $65.3 \pm 24.6 \mathrm{mmol} / \mathrm{mol}$. On average, the participants had a high fear of fall score (using FES-I) of $33.9 \pm 11.8$, with 19 participants reporting $\geq 1$ falls in the past year. The IG and CG did not differ in any of the baseline variables (table 1). An analysis of the baseline data of all recruited participants revealed that the duration of diabetes had a moderate but significant correlation with the history of multiple falls $(r=0.506, p=0.006)$. A similar association was found between the years of DPN and the severity of neuropathy quantified by the average VPT score $(\mathrm{r}=0.407, \mathrm{p}=0.046)$. We also observed a moderate correlation between the CoM sway and the physical component score of the SF-12 $(r=0.515, \mathrm{p}=0.004)$.

\section{Effect of Balance Training on Outcome Measurements} Postural Stability

Table 2 lists the baseline and follow-up values for both groups. On average, the CoM sway area for the IG was 


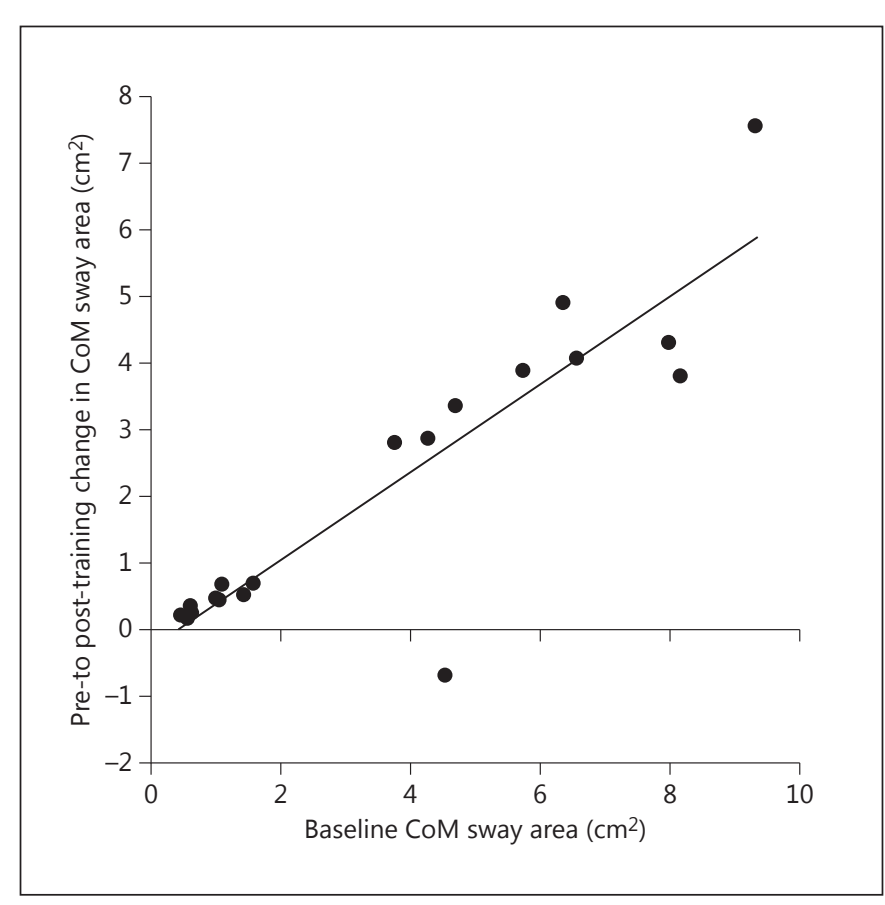

Fig. 3. Improvements in the CoM sway area were significantly associated with poor postural stability at baseline, highlighting that participants with a high risk of fall benefit more from balance training exercises.

reduced significantly by $58.31 \%$ compared to a reduction of $7.8 \%$ in the CG. The IG showed a significant reduction in the ML CoM sway; however, the reduction in their AP CoM sway was nonsignificant. Similarly, significant reductions ( $p=0.017-0.008$ ) were observed for the hip and ankle sway in the IG compared to the CG. The effect size was maximum for the ankle sway $\left(\eta_{\mathrm{p}}{ }^{2}=0.201\right)$. During balance assessment with closed eyes, the IG achieved a reduction in CoM sway of 62.68\%; however, none of the sway components (AP, ML or CoM area) reached significance ( $\mathrm{p}=0.103-0.32$; table 2$)$. The ankle sway was reduced by $58.8 \%$ in the IG compared to $23.03 \%$ in the CG. The hip sway reduction in the IG did not reach significance.

Physical Activity and Quality of Life

Our follow-up measurements revealed a trend for improvement in physical activity in the IG (table 2). On average, the amount of time spent walking increased by $27.26 \%$, and the number of steps taken in $48 \mathrm{~h}$ increased by $27.68 \%$. Neither value reached significance. The SF-12 revealed a significant improvement in the mental score component in the IG compared to the CG. The physical component score $(\mathrm{p}=0.643)$ and FES-I score $(\mathrm{p}=0.305)$ did not change significantly (table 2 ). Furthermore, the balance performance at baseline was significantly associated with the level of improvement in the primary study endpoint (baseline-post-training CoM sway area; $\mathrm{r}=$ $0.888, \mathrm{p}<0.001$; fig. 3 ). No other baseline parameter was associated with pre- and post-training changes in the CoM sway. There was a moderate but significant correlation $(\mathrm{r}=0.550, \mathrm{p}=0.015)$ between the baseline CoM sway and fear of fall in the IG.

\section{Discussion}

The findings of the current study reveal that training in a virtual environment with real-time joint feedback can reduce postural sway in DPN patients. We observed a significant reduction in the total CoM sway of $58.3 \%$ in the IG after 4 weeks of training during balance assessments with open eyes. Our results are in line with recent findings demonstrating improvements in balance and functional tasks from exercise training $[10,28]$. We believe that the dynamic movements of leaning forward and the postural coordination during ankle-reaching training contributed toward these reductions/improvements. Furthermore, the ML CoM sway, which has been more associated with repetitive falls than the AP CoM sway [29], was reduced significantly by about $40 \%$ in the IG. The practice from leaning and shifting weight during the virtual obstaclecrossing task, intended to better control ML body movements, reflects these improvements [30]. Similar benefits of weight-shifting exercises on the postural sway have been reported in the literature [28]. The current study, however, took a step forward and implemented a tailored exercise regime that was specifically intended for patients with impaired joint perception and strength. Given the impaired sense of the joint position, reduced ankle muscle strength $[4,7]$ and fall risk in DPN patients, the exercise training with crossing virtual obstacles, which practices shifting weight, may reduce the number of future falls in people with diabetes. It is also speculative whether visual feedback from joint motion during motor learning enhanced the integration of somatosensory feedback from visual and auditory senses [31, 32].

The secondary outcome (daily physical activities) demonstrated a trend of improvement. Both the time spent walking and the number of steps in $48 \mathrm{~h}$ increased in the IG. Even though nonsignificant improvements were observed, we speculate that improvements in the postural coordination and joint mobility after the train- 
ing contributed to an increased confidence toward a higher level of everyday activities. Previous studies have reported similar effects of exercise on improvements in physical activity $[33,34]$. Besides the postural improvements, it appears that the training had an antidepressant effect on patients based on the changes in their mental component scores of the SF- 12 .

Our findings are supported by the literature, reporting improvements in balance from virtual-reality interactions [21, 28] and auxiliary sensory cues [35]. However, the benefits of visual feedback from joint motion during exercise training on postural control need further exploration. Exergaming approaches have been demonstrated to be feasible and beneficial for older adults [13, $36,37]$; however, there is still room for improvement. Commercially available platform-based (e.g. Nintendo Wii Balance Board) or camera-based systems (Microsoft Kinect) used for exercise training are limited to either narrow force platforms, which are not ideal for individuals with diabetes due to their increased body weight and limited joint mobility, or require a continuous unobstructed line of sight $[13,36,38]$. Additionally, some of the commercial exergaming devices detect only the movement of the controller and do not necessarily require participants to exert whole-body movements, which might not be ideal adaptive approaches for rehabilitation $[14,22]$. Furthermore, the options available to tailor the game for specific population groups or individuals are limited, as visual feedback is intended for game or fun purposes only $[39,40]$ rather than accuracy of performance or feedback on motor errors that enhances learning. Biomechanical validation studies have also reported limited accuracies of such systems [41]. A tailored exercise paradigm specifically for people with DPN has been implemented in the current study, where, besides visual feedback from joint motion, the level of difficulty can be altered over time in order to meet the capabilities of patients and maintain a higher level of motivation and indulgence.

\section{Limitations and Future Research}

We recognize that our sample size is small, and we had relatively few weeks of training. However, the significant improvement in the clinical outcome of balance highlights the effectiveness of this innovative training. Second, the CG did not participate in any form of exercise and did not visit the clinics twice a week, which may have slightly affected the findings. Finally, we did not explore the long-term effect of exercise on improvements in postural balance. Subsequent research with a follow-up at 3-6 months could help to establish whether balance training-induced improvements are retained. Future randomized controlled trials implementing sensor-based exercise in diabetes are warranted with a larger pool of patients and with longer training periods of 8-12 weeks to evaluate its effectiveness for other clinical outcomes including falls, gait and daily physical activities.

\section{Conclusions}

The results of this study suggest that exercise training through an interactive virtual interface with real-time visual feedback from the lower extremity joints can significantly improve postural balance in people with DPN which, in turn, may reduce the risk of fall. Home-based training and its long-term effects should be explored in future research studies.

\section{Acknowledgements}

The study was supported in part by the Qatar National Research Foundation (NPRP-4-1025-3-276). The content is solely the responsibility of the authors and does not necessarily represent the official views of the Qatar National Research Foundation.

\section{Disclosure Statement}

The authors have no conflicts of interest.

\section{References}

Boulton AJ: Management of diabetic peripheral neuropathy. Clin Diabetes 2005;23:9-15.

-2 Armstrong DG, Lavery LA: Diabetic foot ulcers: prevention, diagnosis and classification. Am Fam Physician 1998;57:1325-1332, 1337-1338

3 Levin ME: Diabetes and peripheral neuropathy. Diabetes Care 1998;21:1.

4 Gutierrez EM, et al: Mild diabetic neuropathy affects ankle motor function. Clin Biomech 2001; 16:522-528.
5 Morrison S, et al: Relation between risk of falling and postural sway complexity in diabetes. Gait Posture 2012;35:662-668.

6 Richardson JK, Hurvitz EA: Peripheral neuropathy: a true risk factor for falls. J Gerontol A Biol Sci Med Sci 1995;50:M211-M215.

7 Simoneau GG, et al: Diabetic sensory neuropathy effect on ankle joint movement perception. Arch Phys Med Rehabil 1996;77:453460. 
8 Cavanagh $\mathrm{P}$, et al: Problems with gait and posture in neuropathic patients with insulin-dependent diabetes mellitus. Diabet Med 1992; 9:469-474.

$>9$ Bongaerts BW, et al: Older subjects with diabetes and prediabetes are frequently unaware of having distal sensorimotor polyneuropathy: the KORA F4 study. Diabetes Care 2013; 36:1141-1146.

10 Allet L, et al: The gait and balance of patients with diabetes can be improved: a randomised controlled trial. Diabetologia 2010;53:458466.

11 Morrison S, et al: Balance training reduces falls risk in older individuals with type 2 diabetes. Diabetes Care 2010;33:748-750.

12 Katoulis EC, Boulton AJ, Raptis SA: The role of diabetic neuropathy and high plantar pressures in the pathogenesis of foot ulceration. Horm Metab Res 1996;28:159-164.

13 Agmon M, et al: A pilot study of Wii Fit exergames to improve balance in older adults. J Geriatr Phys Ther 2011;34:161-167.

14 Tanaka K, et al: A comparison of exergaming interfaces for use in rehabilitation programs and research. Loading 2012;6:69-81.

15 Bisson E, et al: Functional balance and dualtask reaction times in older adults are improved by virtual reality and biofeedback training. Cyberpsychol Behav 2007;10:16-23.

16 Cross ES, et al: Sensitivity of the action observation network to physical and observational learning. Cereb Cortex 2009;19:315-326.

17 Boulton AJ, et al: Diabetic neuropathies: a statement by the American Diabetes Association. Diabetes Care 2005;28:956-962.

18 Najafi B, et al: Assessing postural control and postural control strategy in diabetes patients using innovative and wearable technology. J Diabetes Sci Technol 2010;4:780-791.

19 Favre J, et al: Ambulatory measurement of 3D knee joint angle. J Biomech 2008;41:1029_ 1035.

20 Smith MA, Ghazizadeh A, Shadmehr R: Interacting adaptive processes with different timescales underlie short-term motor learning. PLoS Biol 2006;4:e179.
21 Grewal GS, et al: Balance rehabilitation: promoting the role of virtual reality in patients with diabetic peripheral neuropathy. J Am Podiatr Med Assoc 2013;103:498-507.

22 Anderson F, Annett M, Bischof WF: Lean on Wii: physical rehabilitation with virtual reality Wii peripherals. Stud Health Technol Inform 2010;154:229-234.

23 Shabbott BA, Sainburg RL: Learning a visuomotor rotation: simultaneous visual and proprioceptive information is crucial for visuo motor remapping. Exp Brain Res 2010;203: 75-87.

24 Grewal G, et al: Virtualizing the assessment: a novel pragmatic paradigm to evaluate lower extremity joint perception in diabetes. Gerontology 2012;10:10.

25 Najafi B, Armstrong DG, Mohler J: Novel wearable technology for assessing spontaneous daily physical activity and risk of falling in older adults with diabetes. J Diabetes Sci Technol 2013;7:1147-1160.

26 Najafi B, et al: Game-Based Guided Exercise: Using an Avatar with Real-Time Feedback to Improve Postural Stability in Diabetic Peripheral Neuropathy. San Francisco, American Diabetes Association, 2014.

27 Cohen J: Statistical Power Analysis for the Behavioral Sciences. Hillsdale, Lawrence Erlbaum Associates, 1997.

28 Lee S, Shin S: Effectiveness of virtual reality using video gaming technology in elderly adults with diabetes mellitus. Diabetes Technol Ther 2013;15:489-496.

29 Maki BE, Holliday PJ, Topper AK: A prospective study of postural balance and risk of falling in an ambulatory and independent elderly population. J Gerontol 1994;49:M72-M84.

30 Chou L-S, et al: Medio-lateral motion of the center of mass during obstacle crossing distinguishes elderly individuals with imbalance. Gait Post 2003;18:125-133.

31 Westlake KP, Culham EG: Sensory-specific balance training in older adults: effect on proprioceptive reintegration and cognitive demands. Phys Ther 2007;87:1274-1283.
32 Westlake KP, Wu Y, Culham EG: Sensoryspecific balance training in older adults: effect on position, movement, and velocity sense at the ankle. Phys Ther 2007;87:560-568.

33 Hauer K, et al: Physical training improves motor performance in people with dementia: a randomized controlled trial. J Am Geriatr Soc 2012;60:8-15.

34 Mostert S, Kesselring J: Effects of a short-term exercise training program on aerobic fitness, fatigue, health perception and activity level of subjects with multiple sclerosis. Mult Scler 2002;8:161-168.

35 Rao N, Aruin AS: Auxiliary sensory cues improve automatic postural responses in individuals with diabetic neuropathy. Neurorehabil Neural Repair 2011;25:110-117.

36 Chang Y-J, Chen S-F, Huang J-D: A kinectbased system for physical rehabilitation: a pilot study for young adults with motor disabilities. Res Dev Disabil 2011;32:2566-2570.

37 Saposnik G, et al: Effectiveness of virtual reality using Wii gaming technology in stroke rehabilitation: a pilot randomized clinical trial and proof of principle. Stroke 2010;41:14771484.

38 Lange B, et al: Development and evaluation of low cost game-based balance rehabilitation tool using the Microsoft Kinect sensor; in Engineering in Medicine and Biology Society, EMBC, 2011 Annual International Conference of the IEEE. Boston, IEEE, 2011.

39 Hsu JK, et al: A 'Wii' bit of fun: the effects of adding Nintendo $\mathrm{Wii}{ }^{\circledR}$ Bowling to a standard exercise regimen for residents of long-term care with upper extremity dysfunction. Physiother Theory Pract 2011;27:185-193.

40 Wollersheim D, et al: Physical and psychosocial effects of Wii video game use among older women. Int J Emerg Technol Soc 2010;8: 85-98.

41 Fernandez-Baena A, Susín A, Lligadas X: Biomechanical validation of upper-body and lower-body joint movements of kinect motion capture data for rehabilitation treatments; in 4th International Conference on Intelligent Networking and Collaborative Systems (INCoS 2012). Bucharest, IEEE, 2012. 